\title{
Design and Application of Offset-Free Model Predictive Control Disturbance Observation Method
}

\author{
Xue Wang, Baocang Ding, Xin Yang, and Zhaohong Ye \\ College of Automation, Chongqing University, Chongqing 400044, China \\ Correspondence should be addressed to Xue Wang; wangxue052@163.com
}

Received 12 October 2015; Revised 19 March 2016; Accepted 5 April 2016

Academic Editor: Yang Shi

Copyright (C) 2016 Xue Wang et al. This is an open access article distributed under the Creative Commons Attribution License, which permits unrestricted use, distribution, and reproduction in any medium, provided the original work is properly cited.

\begin{abstract}
Model predictive control (MPC) with its lower request to the mathematical model, excellent control performance, and convenience online calculation has developed into a very important subdiscipline with rich theory foundation and practical application. However, unmeasurable disturbance is widespread in industrial processes, which is difficult to deal with directly at present. In most of the implemented MPC strategies, the method of incorporating a constant output disturbance into the process model is introduced to solve this problem, but it fails to achieve offset-free control once the unmeasured disturbances access the process. Based on the Kalman filter theory, the problem is solved by using a more general disturbance model which is superior to the constant output disturbance model. This paper presents the necessary conditions for offset-free model predictive control based on the model. By applying disturbance model, the unmeasurable disturbance vectors are augmented as the states of control system, and the Kalman filer is used to estimate unmeasurable disturbance and its effect on the output. Then, the dynamic matrix control (DMC) algorithm is improved by utilizing the feed-forward compensation control strategy with the disturbance estimated.
\end{abstract}

\section{Introduction}

Model predictive control (MPC) is a control algorithm developed in the process of industrial production, for it can deal with physical constraints and linear multivariable systems flexibly in an online optimal way [1], due to the adoption of multistep prediction, receding optimization, and feedback correction control strategy. Therefore, it has good robustness and low requirements on the accuracy of the model and can deal with time-varying time delay characteristics of the industrial process; the input constraints on the output characteristics, especially, make it widely recognized in the industry and widely used in the field of petroleum and chemical process control. In the coal process industry, coals are various in batches and quality, and their exothermic characteristics are completely different. Since the exothermic characteristic is unmeasurable, it can be regarded as unmeasurable disturbance vector. The production process of coal for methanol is the following: the coal is gasified firstly, and it is handled in the transformation of the process gas and process gas purification; then the process gas is synthesized and the methanol distillation is carried out finally. In this process, if the quality of coal is good (high purity), control parameter (methanol) is stable; that is, the setting value is equal to the actual value; the operation fluctuation is very small and the control effect is good. But if the coal is in bad quality, the actual cannot reach the setting value in the production process and the system will produce fluctuations. Obviously, the unmeasurable disturbance widely exists in the process of industrial production. In order to overcome the disturbance and realize the offsetfree control in industrial processes, the observer is used to measure the unmeasurable disturbance vectors.

Although MPC has many advantages compared to PID control, a weakness still exists: when the control system is influenced by unmeasurable disturbance or modeling errors, and the closed-loop system cannot achieve the expected control performance. In fact, the dynamic matrix control (DMC) has certain limitation. Firstly, the feedback correction is obtained by assuming that there exists a constant output disturbance. Although this method can be done without static error control and is easy to achieve, there is the lack of the advanced estimation for the dynamic characteristics of unmeasurable disturbance, so the effect of disturbance cannot be eased. Secondly, achieving the offset-free control in 
DMC needs an enough accurate system model even though DMC has the characteristics of multistep prediction and receding optimization. Therefore, the modified DMC makes a great difference in industrial production. The authors in [2] design an online identification unmeasurable disturbance DMC controller; this DMC has improved the antijamming performance. Reference [3] discusses the statistical features of process noise and measured noise in unknown circumstances. With the closed-loop monitoring data and output disturbance model, using the Kalman filter online estimates the unmeasurable disturbance to realize the offset-free control in MPC. In [4], a kind of online tuning of feedback corrective coefficient of the MPC algorithm is proposed corresponding to the unmeasurable disturbance. On the other hand, the authors in [5] present a new algorithm for design of MPC with guaranteed stability and constraint satisfaction for linear constrained systems. More recently, the design and implementation of a robust multivariable MPC on a quadruple tanks system is addressed in [6]; the objective of this paper to realize offset-free control of the process in the presence of constraints and model mismatch; model mismatch is compensated by augmented state disturbances.

In addition to all the methods mentioned above, aiming at the shortcomings of the existing DMC algorithms, we introduce a better performance than the constant output disturbance model which is a more general disturbance model, and an online estimation unmeasurable disturbance Kalman filter is designed. Meanwhile, the offset-free sufficient conditions of modified DMC are shown based on the disturbance model. A simulation example for the continuous stirred tank reactor (CSTR) in industrial production illustrates the effectiveness of the improved approach.

\section{Disturbance Modeling Method of Offset-Free Predictive Control}

The approach to eliminate the steady-state error is achieved by assuming that the plant is influenced by the step disturbance of constant value and augmenting disturbance into the process model. It is usually assumed that the disturbance is constant, which is estimated based on measurable variables; then the influence of the controlled variable is eliminated through changing the steady-state object of the controller. Although this approach avoids the limitation of the saturation factors, it is required to design interference model and estimate the disturbance [1].

2.1. Modeling of Unmeasurable Disturbance. If the unmeasurable disturbance or modeling error exists in the control system, the closed-loop can never realize offset-free control. It is necessary to find a method to eliminate the steady-state error under the circumstance of unmeasured disturbance, which is the key to achieve the offset-free control.

Consider the linear time-invariant discrete system:

$$
\begin{aligned}
x(k+1) & =A x(k)+B u(k), \\
y(k) & =C x(k),
\end{aligned}
$$

where $k$ is the sampling time, $x(k) \in R^{n}$ is the state vector, $y(k) \in R^{l}$ is the observation signal of the state, and $u(k) \epsilon$ $R^{p}$ is the input, respectively. It is assumed that $(A, B)$ are controllable and $(C, A)$ are measurable:

$$
\begin{aligned}
{\left[\begin{array}{c}
x(k+1) \\
d(k+1)
\end{array}\right] } & =\left[\begin{array}{cc}
A & C_{d} \\
0 & I
\end{array}\right]\left[\begin{array}{l}
x(k) \\
d(k)
\end{array}\right] u(k), \\
y(k) & =\left[\begin{array}{ll}
C & 0
\end{array}\right]\left[\begin{array}{l}
x(k) \\
d(k)
\end{array}\right]
\end{aligned}
$$

where $d(k) \in R^{s_{d}}$ is constant and $s_{d}$ is the number of augmented disturbance states and determines the impact of disturbance on the output.

In this framework, the input disturbance can be conveniently described, that is, $C_{d}=B$, and the input disturbance model can be established.

The performance of the closed-loop control system directly depends on the accuracy of the disturbance model characterizing the actual system. Therefore, in order to improve the control performance, a disturbance model, which is matched with the entering process, is adopted so as to maximize the accuracy of the disturbance model. The model is shown as follows:

$$
\begin{gathered}
{\left[\begin{array}{c}
x(k+1) \\
d(k+1) \\
p(k+1)
\end{array}\right]=\left[\begin{array}{ccc}
A & C_{d} & 0 \\
0 & I & 0 \\
0 & 0 & I
\end{array}\right]\left[\begin{array}{l}
x(k) \\
d(k) \\
p(k)
\end{array}\right]+\left[\begin{array}{l}
B \\
0 \\
0
\end{array}\right] u(k),} \\
y(k)=\left[\begin{array}{lll}
C & 0 & C_{p}
\end{array}\right]\left[\begin{array}{ll}
x(k) d(k) & p(k)
\end{array}\right]^{T} .
\end{gathered}
$$

By omitting sampling time $k$ temporarily, denoting

$$
\begin{aligned}
& \bar{x}=\left[\begin{array}{l}
x \\
d \\
p
\end{array}\right], \\
& \bar{A}=\left[\begin{array}{lll}
A & C_{d} & 0 \\
0 & I & 0 \\
0 & 0 & I
\end{array}\right], \\
& \bar{B}=\left[\begin{array}{l}
B \\
0 \\
0
\end{array}\right], \\
& \bar{C}=\left[\begin{array}{lll}
C & 0 & C_{p}
\end{array}\right],
\end{aligned}
$$

one can obtain the disturbance model:

$$
\begin{aligned}
\bar{x}(k+1) & =\bar{A} \bar{x}(k)+\bar{B} u(k), \\
y(k) & =\bar{C} \bar{x}(k) .
\end{aligned}
$$

The state disturbance and output disturbance exist in the model simultaneously. If $C_{d}=B$, it includes input disturbance and output disturbance. Thus, the disturbance model shown in formula (5) can maximize the accuracy of the actual disturbance system. 
2.2. Realization Conditions of Offset-Free Predictive Control. From the augmented system state estimator in (5), the following steady-state estimation can be obtained:

$$
\begin{aligned}
& \hat{x}_{\infty}= A \widehat{x}_{\infty}+B u_{\infty}+C_{d} \widehat{d}_{\infty} \\
&+K_{1}\left(y_{\infty}-C \widehat{x}_{\infty}-C_{p} \widehat{p}_{\infty}\right), \\
& {\left[\begin{array}{c}
\hat{d}_{\infty} \\
\widehat{p}_{\infty}
\end{array}\right]=} {\left[\begin{array}{l}
\hat{d}_{\infty} \\
\widehat{p}_{\infty}
\end{array}\right]+K_{n}\left(y_{\infty}-C \widehat{x}_{\infty}-C_{p} \widehat{p}_{\infty}\right), } \\
& K_{n}=\left[\begin{array}{c}
K_{2} \\
K_{3}
\end{array}\right],
\end{aligned}
$$

where $u_{\infty}$ is the steady-state value calculated by the controller input and $y_{\infty}$ is measurable steady-state process output.

Due to the fact that the augmented system is measurable and $S_{d}+S_{p}=L, K_{n}$ is a nonsingular matrix. From (5) and (6), full rank means that

$$
\begin{aligned}
& y_{\infty}=C \widehat{x}_{\infty}+C_{p} \widehat{p}_{\infty}, \\
& \widehat{x}_{\infty}=A \widehat{x}_{\infty}+B u_{\infty}+C_{d} \widehat{d}_{\infty} .
\end{aligned}
$$

With regard to the steady-state control input $u_{s}$ and state target vector $x_{s}$, the following equation holds:

$$
x_{s}=A x_{s}+B u_{s}+C_{d} \widehat{d}_{\infty} .
$$

Equation (8) minus (9) satisfies

$$
\widehat{x}_{\infty}-x_{s}=A\left(\hat{x}_{\infty}-x_{s}\right)+B\left(u_{\infty}-u_{s}\right) \text {. }
$$

It can be seen that the controller in steady-state is unconstrained; therefore, the control input can be calculated by the method of infinite horizon controller:

$$
u_{\infty}-u_{s}=-K_{x}\left(\widehat{x}_{\infty}-u_{s}\right) .
$$

The nominal stability of the feedback gain implies that the matrix $A-B K_{x}$ is stable; thus, the only solution is $\widehat{x}_{\infty}-x_{s}$. When the state target vector is $x_{s}$ and the estimated value of the steady-state output disturbance is $\widehat{p}_{\infty}$, the reachable output objective is

$$
y_{t}^{s}=C x_{s}-C_{p} \widehat{p}_{\infty}
$$

where $y_{t}^{s}$ is a reachable steady-state output objective. Consider

$$
y_{\infty}-y_{t}^{s}=C\left(\widehat{x}_{\infty}-x_{s}\right)=0
$$

It indicates $y_{\infty}=y_{t}^{s}$ and the realization of offset-free control.

The following method will be used to judge whether the augmented matrices $(\bar{C}, \bar{A})$ are measurable and the Hantus theorem is the theoretical basis of the method.

Hantus theorem points out the sufficient and necessary condition of measurable augmented system in [7] as follows:

$$
\operatorname{rank}\left[\begin{array}{c}
\lambda I-\bar{A} \\
\bar{C}
\end{array}\right]=n+s_{d}+s_{p}, \quad \forall \lambda \in C, \quad|\lambda| \geq 1
$$

It only needs to check $\lambda=\operatorname{eig}(A), \lambda \geq 1$, for all $\lambda \in C$, and it is the only situation of the matrix nonsingular, for all $\lambda \geq 1, \lambda \in C$. By substituting the augmented system matrix into the Hantus condition equation, if and only if

$$
\begin{gathered}
{\left[\begin{array}{ccc}
\lambda I-A & -C_{d} & 0 \\
0 & (\lambda-I) I_{s d} & 0 \\
0 & 0 & (\lambda-I) I_{s p} \\
C & 0 & C_{d}
\end{array}\right]\left[\begin{array}{l}
x \\
d \\
p
\end{array}\right] \neq 0,} \\
\forall\left[\begin{array}{l}
x \\
d \\
p
\end{array}\right] \neq 0, \lambda \in\left\{1, \lambda_{s}^{A}\right\},
\end{gathered}
$$

the augmented system $(\bar{C}, \bar{A})$ is measurable, where $\lambda_{s}^{A}=\{\lambda$ : $\lambda=\operatorname{eig}(A),|\lambda| \geq 1\}$ is unstable eigenvalue in matrix $A$. The upper block with the dimensional $\left(n+s_{d}+s_{p}\right) \times\left(n+s_{d}+s_{p}\right)$ of the matrix is nonsingular when $\lambda \in\left\{1, \lambda_{s}^{A}\right\}$ is satisfied; that is, it only needs to consider these values. Based on the above equation, the measurable conditions of augmented systems $(\bar{C}, \bar{A})$ can be further obtained:

$$
\operatorname{rank}\left[\begin{array}{ccc}
I-A & -C_{d} & 0 \\
C & 0 & C_{p}
\end{array}\right]=n+s_{d}+s_{p}
$$

\section{Multivariable Constrained Offset-Free DMC Algorithm}

DMC is a kind of MPC algorithm based on the step response coefficient model; this paper will present detailed design methods and steps of constrained multivariable DMC algorithm to achieve offset-free control; the improved algorithm not only realizes the offset-free control, but also has the strong ability of disturbance suppression.

From the theory of the DMC algorithm, the estimated $\widehat{d}(k)$ is introduced into prediction and correction link of offset-free DMC algorithm to achieve the feed-forward compensation. According to superposition theorem, the derivation process of multivariable constrained offset-free DMC algorithm is given by the following $[8,9]$.

Step 1. Supposing that only the $j$ th input is affected by the unmeasurable disturbance, the feed-forward compensation control strategy is introduced for the $j$ th input based on the estimation. In this way, the input of the $j$ th input predictive correction link becomes

$$
u_{j}(k)=u_{c, j}(k)+u_{d, j}(k) \text {, }
$$

where $u_{c, j}(k)$ and $u_{d, j}(k)$ are manipulated variable and input produced by unmeasurable disturbance, respectively; the sum of them constitutes the $j$ th input. If other inputs do not 
change and consider the ith input, one can obtain the following based on (16):

$$
\begin{aligned}
& y_{i j, 0}(k+\bar{i} \mid k-1) \\
& =s_{i j, \bar{i}+1} u_{j}(k-1)+\sum_{\bar{j}=2}^{N-\bar{i}}\left(s_{i j, \bar{i}+\bar{j}}-s_{i j, \bar{i}+\bar{j}-1}\right) u_{j}(k-\bar{j}) \\
& =s_{i j, \bar{i}+1}\left(u_{c, j}(k-1)+u_{d, j}(k+1)\right) \\
& +\sum_{\bar{j}=2}^{N-\bar{i}}\left(s_{i j, \bar{i}+\bar{j}}-s_{i j, \bar{i}+\bar{j}-1}\right)\left(u_{c, j}(k-\bar{j})+u_{d, j}(k-\bar{j})\right) \\
& =s_{i j, \bar{i}+1} u_{c, j}(k-1)+\sum_{\bar{j}=2}^{N-\bar{i}}\left(s_{i j, \bar{i}+\bar{j}}-s_{i j, \bar{i}+\bar{j}-1}\right) u_{c, j}(k-\bar{j}) \\
& +s_{i j, \bar{i}+1} u_{d, j}(k-1) \\
& \quad+\sum_{\bar{j}=2}^{N-\bar{i}}\left(s_{i j, \bar{i}+\bar{j}}-s_{i j, \bar{i}+\bar{j}-1}\right) u_{d, j}(k-\bar{j}), \\
&
\end{aligned}
$$

Then the following equation is established:

$$
\begin{aligned}
y_{i j, 0}(k+\bar{i} \mid k-1)= & y_{i j, 0 c}(k+\bar{i} \mid k-1) \\
& +y_{i j, 0} d(k+\bar{i} \mid k-1), \\
& \quad \bar{i} \in\{1,2, \ldots, P\},
\end{aligned}
$$

in which $y_{i j, 0 c}(k+\bar{i} \mid k-1)$ and $y_{i j, 0 d}(k+\bar{i} \mid k-1)$ are output predictive value of manipulated variable and produced by unmeasurable disturbance under the assumption that the control inputs are invariant at current and future time.

Using the feed-forward compensation control strategy with (18), there is

$$
\tilde{u}_{j, p}(k)=\tilde{u}_{c j, p}(k)+\widetilde{u}_{d j, p}(k),
$$

where

$$
\begin{aligned}
& \tilde{u}_{c j, p}(k) \\
& =\left[u_{c, j}(k-1)+u_{c, j}(k-2), \ldots, u_{c, j}(k-N+1)\right]^{T}, \\
& \tilde{u}_{d j, p}(k) \\
& =\left[u_{d, j}(k-1)+u_{d, j}(k-2), \ldots, u_{d, j}(k-N+1)\right]^{T} .
\end{aligned}
$$

It is rewritten in the form of vector; there exists

$$
\begin{aligned}
\tilde{y}_{i j, 0}(k \mid k-1) & =A_{i j, p} \tilde{u}_{j, p}(k) \\
& =A_{i j, p} \tilde{u}_{c j, p}(k)+A_{i j, p} \tilde{u}_{d j, p}(k) .
\end{aligned}
$$

Step 2. If all of the inputs are likely to be affected by the unmeasurable disturbance, then adopting the feed-forward compensation control strategy and considering the $i$ th output [10], the equation is given by using superposition theorem:

$$
\begin{aligned}
y_{i, 0}( & +\bar{i} \mid k-1) \\
= & y_{i 1,0}(k+\bar{i} \mid k-1)+y_{i 2,0}(k+\bar{i} \mid k-1)+\cdots \\
& +y_{i m, 0}(k+\bar{i} \mid k-1) \\
= & s_{i 1, \bar{i}+1}\left(u_{c, 1}(k-1)+u_{d, 1}(k-1)\right) \\
& +\sum_{\bar{j}=2}^{N-\bar{i}}\left(s_{i 1, \bar{i}+\bar{j}}-s_{i 1, \bar{i}+\bar{j}-1}\right)\left(u_{c, 1}(k-\bar{j})\right) \\
& +\left(u_{d, 1}(k-\bar{j})\right)+s_{i 2, \bar{i}+1}\left(u_{c, 2}(k-1)\right) \\
& +\left(u_{d, 2}(k-1)\right) \\
& +\sum_{\bar{j}=2}^{N-\bar{i}}\left(s_{i 2, \bar{i}+\bar{j}}-s_{i 2, \bar{i}+\bar{j}-1}\right)\left(u_{c, 2}(k-\bar{j})\right) \\
+ & \left(u_{d, 2}(k-\bar{j})\right)+\cdots+s_{i m, \bar{i}+1}\left(u_{c, m}(k-1)\right) \\
+ & \left(u_{d, m}(k-1)\right) \\
& +\sum_{\bar{j}=2}^{N-\bar{i}}\left(s_{i m, \bar{i}+\bar{j}}-s_{i m, \bar{i}+\bar{j}-1}\right)\left(u_{c, m}(k-\bar{j})\right) \\
+ & (k-\bar{j})) \quad u_{d, m}(k, 2, \ldots, P\} . \\
&
\end{aligned}
$$

The vector form is

$$
\begin{aligned}
\tilde{y}_{i, 0}(k \mid k-1) & =\sum_{j=1}^{m} A_{i j, p} \tilde{u}_{j, p}(k) \\
& =\sum_{j=1}^{m} A_{i j, p} \tilde{u}_{j, p}(k)+\tilde{u}_{d j, p}(k) .
\end{aligned}
$$

Similar to (20), let

$$
\begin{aligned}
& \tilde{y}_{c i, 0}(k \mid k-1)=\sum_{j=1}^{m} A_{i j, p} \tilde{u}_{c j, p}(k), \\
& \tilde{y}_{d i, 0}(k \mid k-1)=\sum_{j=1}^{m} A_{i j, p} \tilde{u}_{d j, p}(k) .
\end{aligned}
$$

The following can be obtained:

$$
\tilde{y}_{i, 0}(k \mid k-1)=\tilde{y}_{c i, 0}(k \mid k-1)+\tilde{y}_{d i, 0}(k \mid k-1) .
$$

Thus, all the inputs are compensated in the way of feedforward and all the outputs are considered; each $\tilde{y}_{i, 0}(k \mid k-$ $1)$ in $Y_{0}(k \mid k-1)$ is composed of $\widetilde{y}_{c i, 0}(k \mid k-1)$ and $\widetilde{y}_{c i, 0}(k \mid$ $k-1)$ which are output predictive value of manipulated variable and produced by unmeasurable disturbance under the assumption that the control inputs are invariant at current and future time. 
Step 3. Consider the ith input, and it can be corrected based on the predictive error.

Firstly, let $\bar{i}=0$, and the output predictive value of $i$ th output at time $k$ predicted at time $k-1$ is

$$
\begin{aligned}
\tilde{y}_{i, 0} & (k \mid k-1)=y_{i 1,0}(k \mid k-1)+y_{i 2,0}(k \mid k-1) \\
& +\cdots+y_{i m, 0}(k \mid k-1) \\
& =s_{i 1,1}\left(u_{c, 1}(k-1)+u_{d, 1}(k-1)\right) \\
& +\sum_{\bar{j}=2}^{N}\left(s_{i 1, \bar{j}}-s_{i 1, \bar{j}-1}\right)\left(u_{c, 1}(k-\bar{j})+u_{d, 1}(k-\bar{j})\right) \\
+ & s_{i 2,1}\left(u_{c, 2}(k-1)+u_{d, 2}(k-1)\right) \\
+ & \sum_{\bar{j}=2}^{N}\left(s_{i 2, \bar{j}}-s_{i 2, \bar{j}-1}\right)\left(u_{c, 2}(k-\bar{j})+u_{d, 2}(k-\bar{j})\right) \\
+ & \cdots+s_{i m, 1}\left(u_{c, m}(k-1)+u_{d, m}(k-1)\right) \\
& +\sum_{\bar{j}=2}^{N}\left(s_{i m, \bar{j}}-s_{i m, \bar{j}-1}\right)\left(u_{c, m}(k-\bar{j})+u_{d, m}(k-\bar{j})\right) .
\end{aligned}
$$

The vector form of (27) is written as

$$
\begin{aligned}
y_{i, 0}(k \mid k-1) & =\sum_{j=1}^{m} \bar{A}_{i j, p} \bar{u}_{j, p}(k) \\
& =\sum_{j=1}^{m} \bar{A}_{i j, p} \bar{u}_{c j, p}(k)+\bar{u}_{d j, p}(k),
\end{aligned}
$$

where

$$
\begin{aligned}
& \bar{A}_{i j, p}=\left[\begin{array}{llll}
s_{i j, 1} & s_{i j, 2}-s_{i j, 1} & \cdots & s_{i j, N}-s_{i j, N-1}
\end{array}\right], \\
& \bar{u}_{c j, p}(k)=\bar{u}_{c j, p}(k)+\bar{u}_{d j, p}(k) \text {, } \\
& \bar{u}_{j, p}(k)=\left[u_{j}(k-1), u_{j}(k-2), \ldots, u_{j}(k-N)\right]^{T} \text {, } \\
& \bar{u}_{c j, p}(k) \\
& =\left[u_{c, j}(k-1), u_{c, j}(k-2), \ldots, u_{c, j}(k-N)\right]^{T}, \\
& \bar{u}_{d j, p}(k) \\
& =\left[u_{d, j}(k-1), u_{d, j}(k-2), \ldots, u_{d, j}(k-N)\right]^{T} .
\end{aligned}
$$

Denote

$$
\begin{aligned}
& y_{c i, 0}(k \mid k-1)=\sum_{j=1}^{m} \bar{A}_{i j, p} \bar{u}_{c j, p}(k), \\
& y_{d i, 0}(k \mid k-1)=\sum_{j=1}^{m} \bar{A}_{i j, p} \bar{u}_{d j, p}(k)
\end{aligned}
$$

and we can get

$$
y_{i, 0}(k \mid k-1)=y_{c i, 0}(k \mid k-1)+y_{d i, 0}(k \mid k-1) .
$$

When the system is influenced by the unmeasurable disturbance, the actual system can be seen as $y_{i}(k)=y_{c, i}(k)+$ $y_{d, i}(k)$, where $y_{c, i}(k)$ and $y_{d, i}(k)$ are generated by manipulated variable and unmeasured disturbances, respectively. Through comparing $y_{i, 0}(k \mid k-1)$ with the actual output $y_{i}(k)$, the prediction error is given by

$$
\begin{aligned}
\mathcal{E}(k) & =\left[\begin{array}{c}
\varepsilon_{1}(k) \\
\varepsilon_{2}(k) \\
\vdots \\
\varepsilon_{r}(k)
\end{array}\right]=\left[\begin{array}{c}
y_{1}(k)-y_{1,0}(k k-1) \\
y_{2}(k)-y_{2,0}(k k-1) \\
\vdots \\
y_{r}(k)-y_{r, 0}(k k-1)
\end{array}\right] \\
= & {\left[\begin{array}{c}
y_{c, 1}(k)-y_{c 1,0}(k)+y_{d, 1}(k)-y_{d 1,0}(k) \\
y_{2}(k)-y_{c 2,0}(k)+y_{d, 2}(k)-y_{d 2,0}(k) \\
\vdots \\
y_{r}(k)-y_{c r, 0}(k)+y_{d, r}(k)-y_{d r, 0}(k)
\end{array}\right] . }
\end{aligned}
$$

With correction, it becomes as follows:

$$
\begin{aligned}
& \tilde{y}_{i, 0}(k \mid k)=\tilde{y}_{i, 0}(k \mid k-1)+\sum_{\lambda=1}^{r} \breve{f}_{s \lambda} \varepsilon_{i} \\
& =\tilde{y}_{c i, 0}(k \mid k-1)+\widetilde{y}_{d i, 0}(k \mid k-1) \\
& \quad+\sum_{\lambda=1}^{r} \breve{f}_{s \lambda}\left(\tilde{y}_{c i, 0}(k)-y_{c i, 0}(k)+\tilde{y}_{d, i}(k)-\tilde{y}_{d i, 0}(k)\right) \\
& =\tilde{y}_{c i, 0}(k \mid k-1)+\sum_{\lambda=1}^{r} \breve{f}_{s \lambda}\left(\tilde{y}_{c, i}(k)-\tilde{y}_{c i, 0}(k)\right) \\
& +\tilde{y}_{d i, 0}(k \mid k-1)+\sum_{\lambda=1}^{r} \breve{f}_{s \lambda}\left(\tilde{y}_{d, i}(k)-\tilde{y}_{d i, 0}(k)\right) .
\end{aligned}
$$

Similar to (30), let

$$
\begin{aligned}
& \tilde{y}_{c i, 0}(k \mid k)= \tilde{y}_{c i, 0}(k \mid k-1) \\
&+\sum_{\lambda=1}^{r} \breve{f}_{s \lambda}\left(\widetilde{y}_{c, i}(k)-\tilde{y}_{c i, 0}(k)\right), \\
& \tilde{y}_{d i, 0}(k \mid k)=\tilde{y}_{d i, 0}(k \mid k-1) \\
&+\sum_{\lambda=1}^{r} \breve{f}_{s \lambda}\left(\tilde{y}_{d, i}(k)-\tilde{y}_{d i, 0}(k)\right) .
\end{aligned}
$$

Then one can obtain

$$
\tilde{y}_{i, 0}(k \mid k)=\tilde{y}_{c i, 0}(k \mid k)+\tilde{y}_{d i, 0}(k \mid k),
$$

where $\tilde{y}_{i, 0}(k \mid k)$ is composed of $\tilde{y}_{c i, 0}(k \mid k)$ and $\tilde{y}_{d i, 0}(k \mid k)$, which are generated from manipulated variable; it is assumed that the output prediction value of the control inputs is invariant at current and future time.

Step 4. Consider all of the outputs:

$$
\begin{aligned}
Y_{0}(k \mid k) & =Y_{c, 0}(k \mid k)+Y_{d, 0}(k \mid k), \\
Y_{0}(k \mid k-1) & =Y_{c, 0}(k \mid k-1)+Y_{d, 0}(k \mid k-1),
\end{aligned}
$$


in which

$$
\begin{aligned}
& Y_{c, 0}(k \mid k)=\left[\begin{array}{c}
\tilde{y}_{c 1,0}(k \mid k) \\
\tilde{y}_{c 2,0}(k \mid k) \\
\vdots \\
\tilde{y}_{c r, 0}(k \mid k)
\end{array}\right], \\
& Y_{d, 0}(k \mid k)= {\left[\begin{array}{c}
\tilde{y}_{d 1,0}(k \mid k) \\
\tilde{y}_{d 2,0}(k \mid k) \\
\vdots \\
\tilde{y}_{d r, 0}(k \mid k)
\end{array}\right], } \\
& Y_{c, 0}(k \mid k-1)= {\left[\begin{array}{c}
\tilde{y}_{c 1,0}(k \mid k-1) \\
\tilde{y}_{c 2,0}(k \mid k-1) \\
\vdots \\
\tilde{y}_{c r, 0}(k \mid k-1)
\end{array}\right], } \\
& Y_{d, 0}(k \mid k-1)=\left[\begin{array}{c}
\tilde{y}_{d 1,0}(k \mid k-1) \\
\tilde{y}_{d 2,0}(k \mid k-1) \\
\tilde{y}_{d r, 0}(k \mid k-1)
\end{array}\right] .
\end{aligned}
$$

The real-time control increments are

$$
\begin{aligned}
\Delta u(k) & =D\left(Y_{s}(k)-Y_{0}(k \mid k)\right) \\
& =D\left(Y_{s}(k)-Y_{c, 0}(k \mid k)-Y_{d, 0}(k \mid k)\right) .
\end{aligned}
$$

In the control law of (25), $Y_{d, 0}(k \mid k)$ is produced by unmeasurable disturbance. Thus, the control law considers the effect of the unmeasured disturbance, and this effect in the output is deducted from expectation value in the optimization horizon which constitutes a new expectation value. In order to overcome the disturbance, receding horizon optimization problem with controllable input is introduced.

The above algorithm is based on the uncontrollable input disturbance, and the feed-forward structure of output disturbance can be designed [11] as follows.

Consider that the $i$ th input is not affected by the unmeasurable disturbance $p_{i}(k)$; there exists

$$
y_{i}^{\prime \prime}(k)=y_{i}^{\prime}(k)+p_{i}(k),
$$

where $y_{i}^{\prime}(k)$ is the output without disturbance, which is generated by manipulated variable. If it is influenced by the disturbance $p_{i}(k)$, the disturbance output $y_{i}^{\prime \prime}(k)$ contains $y_{i}^{\prime}(k)$ and $p_{i}(k)$. Owing to the unmeasurable disturbance $p_{i}(k),(38)$ is not used to calculate $y_{i}^{\prime \prime}(k)$, but it can be measured in output directly. Then, using Kalman filter to estimate the value of unmeasurable disturbance $\widehat{p}_{i}(k)$ and deducting $\widehat{p}_{i}(k)$ from $y_{i}^{\prime \prime}(k)$ :

$$
y_{i}(k)=y_{i}^{\prime \prime}(k)-\widehat{p}_{i}(k) .
$$

If $\widehat{p}_{i}(k)=p_{i}(k)$ is satisfied, there is $y_{i}(k)=y_{i}^{\prime}(k)$, it eliminates the unmeasurable disturbance effects $p_{i}(k)$ on the output completely. Certainly, if $\widehat{p}_{i}(k) \neq p_{i}(k)$ is satisfied, the output with $\widehat{p}(k)$ is still superior than the output without disturbance, and the effect of unmeasurable output disturbance is eliminated based on the correction link of the algorithm.

In this way, the perdition error $\varepsilon_{i}(k)$ considers the unmeasurable disturbance. The influence of unmeasurable output disturbance is deducted from the perdition error $\varepsilon_{i}(k)$ in the correction link; it constitutes the new prediction value, which can overcome the disturbance.

Therefore, the design of constrained multivariable offsetfree controller is completed. Not only does the above method has good expansibility and is easily applied to control system, but also it significantly improves the performance of the algorithm [12]. In addition, by applying the linear design method to design the predictive controller, the online calculation of the controller is greatly reduced, which meets the real-time control requirement.

\section{Simulation}

CSTR is widely used in the process of polymer production equipment, and it is the most extensive chemical reactor used in industrial production process.

The dynamic CSTR system can be described as follows:

$$
\begin{aligned}
V \frac{d C_{A}}{d t}= & F\left(C_{A f}-C_{A}\right)-V k_{0} \exp \left(-\frac{E}{R_{g} T_{r}}\right) C_{A}, \\
V_{p} C_{p} \frac{d T_{r}}{d t}= & \rho C_{p} F\left(T_{f}-T_{r}\right)+V(-\Delta H) k_{0} \\
& \times \exp \left(-\frac{E}{R_{g} T_{r}}\right) C_{A} \\
& -U A_{h}\left(T_{r}-T_{c}\right) .
\end{aligned}
$$

The operating points are $y_{0}=x_{0}=(0.2,1.33)$ and $u_{0}=(-0.215,4.51)$. By discretizing the model with sampling period $0.01 \mathrm{~s}$, the corresponding discrete-time model can be rewritten as

$$
\begin{aligned}
x(k+1) & =\bar{A} x(k)+\bar{B} u(k), \\
y(k) & =\bar{C} x(k),
\end{aligned}
$$

where

$$
\begin{aligned}
& \bar{A}=\left[\begin{array}{cccccccc}
0.989 & 150 & 168 & 57 & 0.000248 & 993 & 214 & 342 \\
-0.023 & 19 & 422 & 665 & 0.991 & 49 & 237 & 618
\end{array}\right], \\
& \bar{B} \\
& =\left[\begin{array}{llllllll}
-0.11895 & 296 & 500 & 758 & 0.000248 & 993 & 214 & 342 \\
-0.0148 & 288 & 441 & 874 & 0.00298 & 655 & 519 & 101
\end{array}\right], \\
& \bar{C}=\left[\begin{array}{ll}
1 & 0 \\
0 & 1
\end{array}\right] .
\end{aligned}
$$

According to the designed offset-free controller, the parameter values are the length of model $N=30$; optimization horizon $P=12$; control horizon $M=8$; error weighting 

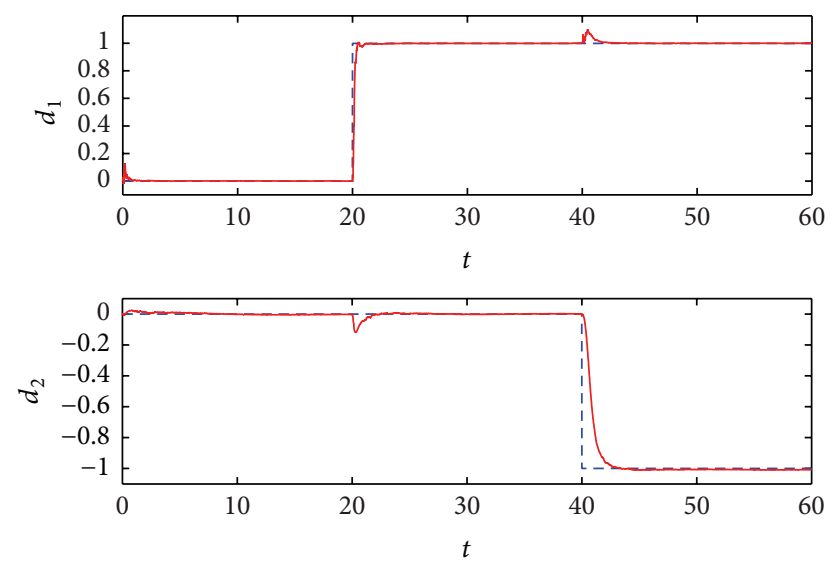

FIGURE 1: True and estimated value of the input disturbance.

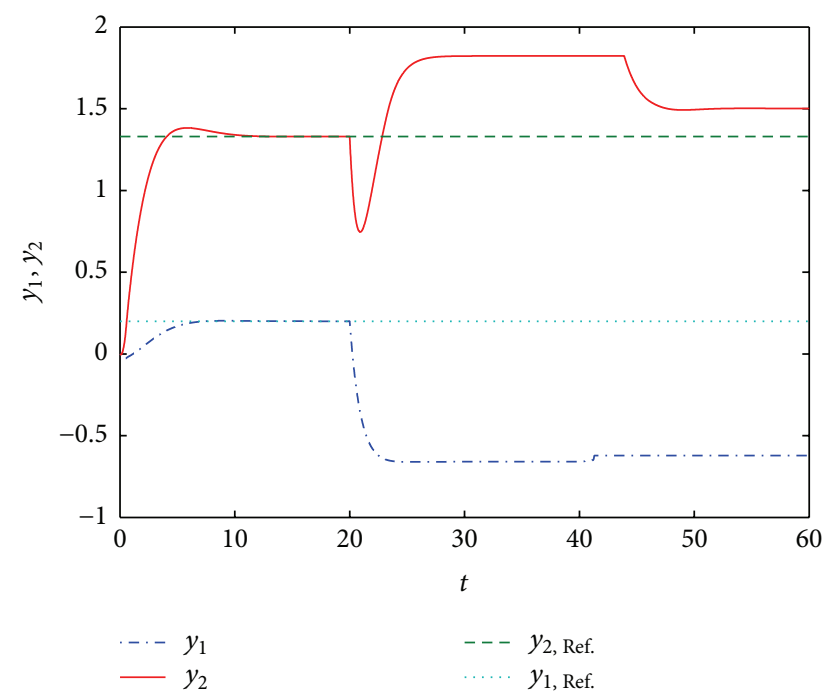

FIGURE 2: Output of algorithm in constrained condition with input disturbance.

matrix $Q=I_{30 \times 30}$; and control weighting matrix $R=6 I_{16 \times 16}$. Hence, the constraint conditions are described as follows.

The situation contains two unmeasurable input disturbances:

$$
\begin{aligned}
-0.5 & <\Delta u_{1}<0.5, \\
-0.25 & <\Delta u_{1}<0.85, \\
-0.5 & <\Delta u_{2}<0.5, \\
0 & <\Delta u_{2}<4.6 .
\end{aligned}
$$

Figure 1 shows that, in the constrained case, filter can also estimate the unmeasured disturbance size.

Figure 2 shows that, after being affected by interference, constrained and unconstrained algorithms are like complete deviations from the expected control objectives, but the interference of outputs $y_{1}$ and $y_{2}$ has smaller impact than unconstrained.

It can be seen that the control increments and inputs in Figures 1 and 2 satisfy constraint conditions, the inputs $u_{1}$ and

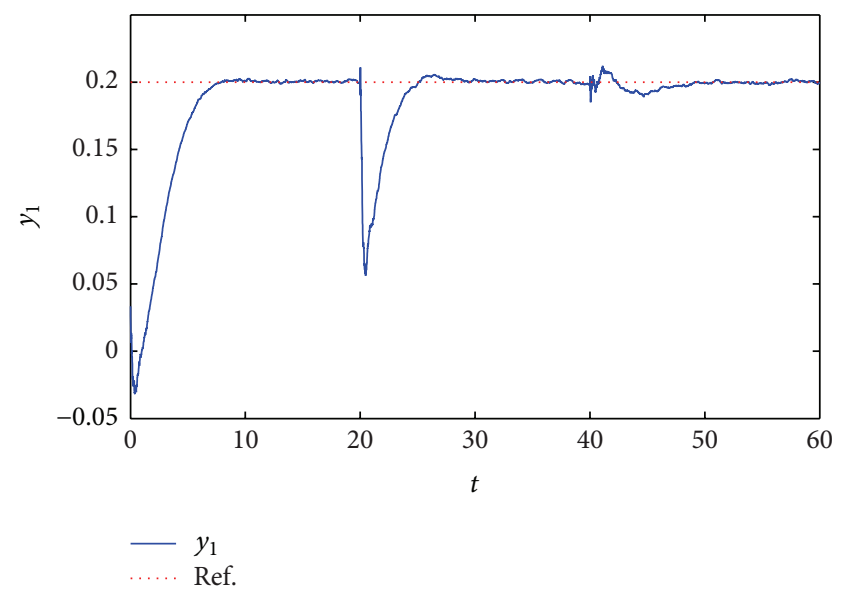

FIGURE 3: Output $y_{1}$ in constrained condition with input disturbance.

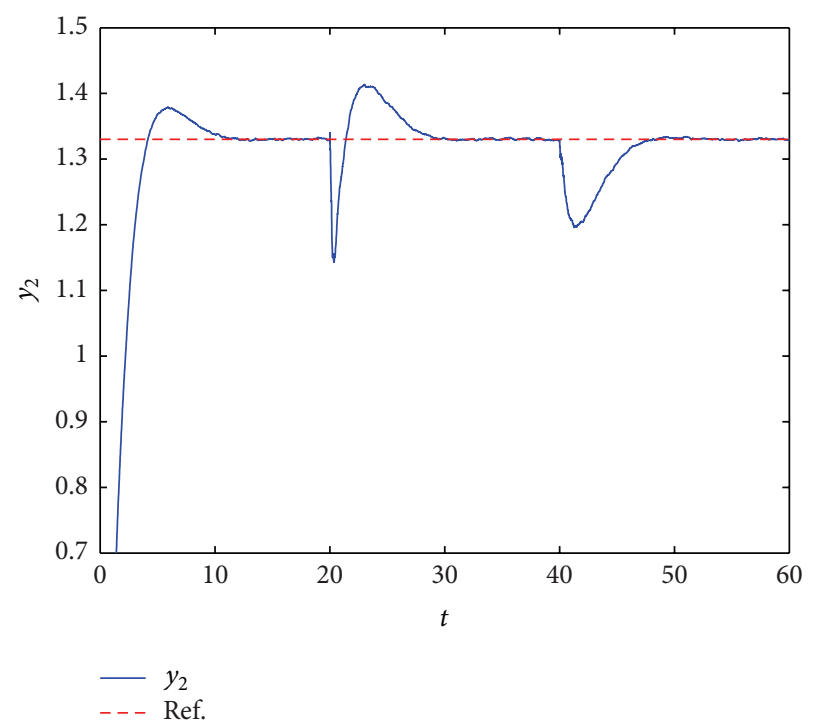

FIGURE 4: Output $y_{2}$ in constrained condition with input disturbance.

$u_{2}$ are more smooth and the shocks are smaller, and the performance is more obvious when the system is in rising time and affected by the unmeasured disturbances. Figure 1 shows that the Kalman filter can also be able to estimate the value of unmeasurable disturbance with constraint conditions. As shown in Figure 2, both of the constrained algorithm and unconstrained algorithm deviate from the expected control objectives when they are affected by the disturbances; however the unconstrained algorithm has less effects for outputs $y_{1}$ and $y_{2}$.

Figures 3 and 4 show that the constrained algorithm has stronger ability of disturbance suppression; it guarantees that the system obtains expected control objective at the effect of disturbances. The physical constrains exist in all control systems, and the unconstrained algorithm is unable to be achieved in the actual system. Therefore, the constrained algorithm has greater practical application value. 


\section{Conclusion}

The difference between the algorithm and the algorithm in constrained condition is that the calculation method of the control increment; the former is obtained by solving the QP problem, and the latter is obtained by solving extreme conditions. Certainly, the computation burden of unconstrained algorithm is lighter than the constrained algorithm. But, in order to obtain the maximum economic benefits with the constrained condition, the constrained algorithm has greater application value. The DMC algorithm has stronger ability of interference suppression; it can guarantee that the system realizes offset-free control in the presence of unmeasurable disturbance. In addition, the predictive controller is designed in the linear method, which reduces the computation burden and guarantees the system performance.

\section{Competing Interests}

The authors declare that they have no competing interests.

\section{References}

[1] S. J. Qin and T. A. Badgwell, "A survey of industrial model predictive control technology," Control Engineering Practice, vol. 11, no. 7, pp. 733-764, 2003.

[2] K. Han, J. Zhao, Y. Zhu, Z. Xu, and J. Qian, "MPC with on-line disturbance model estimation and its application to PTA solvent dehydration tower," Journal of Chemical Industry and Engineering, vol. 59, no. 7, pp. 1657-1664, 2008.

[3] B. J. Odelson and J. B. Rawlings, "Online monitoring of MPC disturbance models using closed-loop data," in Proceedings of the American Control Conference (ACC '03), vol. 3, pp. 27142719, June 2003.

[4] Z.-Y. Ren, H.-H. Shao, and L.-Q. Zhang, "On-line tuning of feedback corrective coefficient of model predictive control," Journal of Shanghai Jiaotong University, vol. 37, no. 6, pp. 922926, 2003.

[5] S. Aghaee, Y. Zakeri, and F. Sheikholeslam, "Offset-free control of constrained linear systems using model predictive control," in Proceedings of the American Control Conference (ACC '12), Montreal, Canada, June 2012.

[6] M. Askari, M. Moghavvemi, H. A. F. Almurib, and K. M. Muttaqi, "Multivariable offset-free model predictive control for quadruple tanks system," IEEE Transactions on Industry Applications, vol. 52, no. 2, pp. 1882-1890, 2015.

[7] P. Lundström, J. H. Lee, M. Morari, and S. Skogestad, "Limitations of dynamic matrix control," Computers and Chemical Engineering, vol. 19, no. 4, pp. 409-421, 1995.

[8] D. W. Clarke, C. Mohtadi, and P. S. Tuffs, "Generalized predictive control-part I. The basic algorithm," Automatica, vol. 23, no. 2, pp. 137-148, 1987.

[9] L.-C. Su and F. Zhu, "Design of a novel omnidirectional stereo vision system," Acta Automatica Sinica, vol. 32, no. 1, pp. 67-72, 2006 (Chinese).

[10] B. W. Bequette, "Non-linear model predictive control: a personal retrospective," The Canadian Journal of Chemical Engineering, vol. 85, no. 4, pp. 408-415, 2007.

[11] B. C. Ding, Modern Predictive Control, CRC Press, Boca Raton, Fla, USA, 2010.
[12] E. Zupan, M. Saje, and D. Zupan, "Quaternion-based dynamics of geometrically nonlinear spatial beams using the Runge-Kutta method," Finite Elements in Analysis and Design, vol. 54, pp. 4860, 2012. 


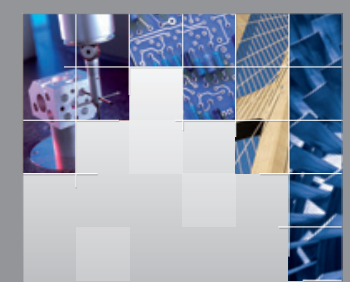

\section{Enfincering}
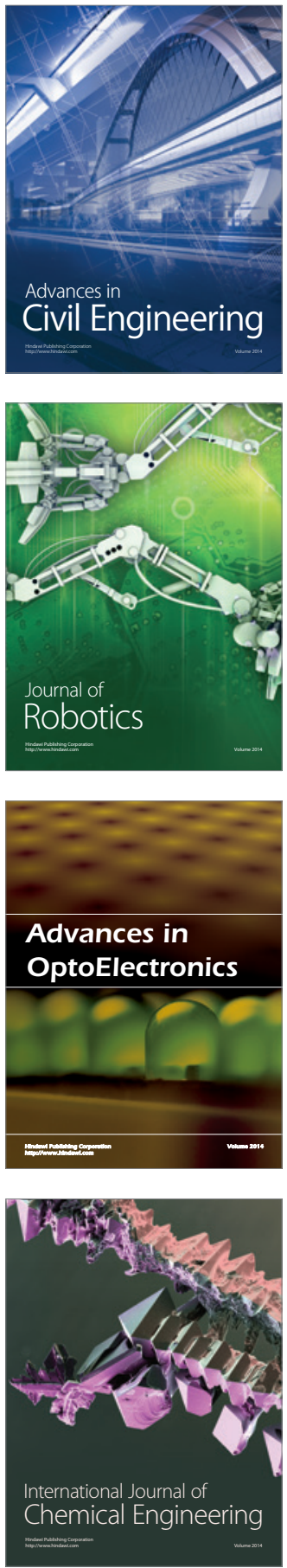

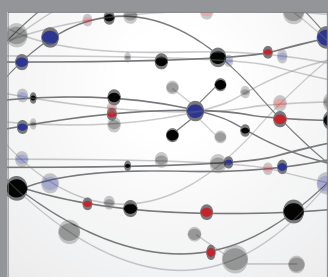

The Scientific World Journal

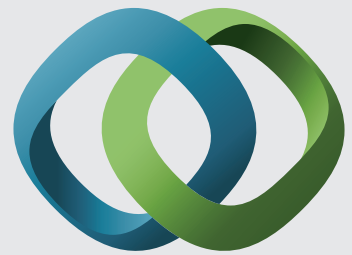

\section{Hindawi}

Submit your manuscripts at

http://www.hindawi.com
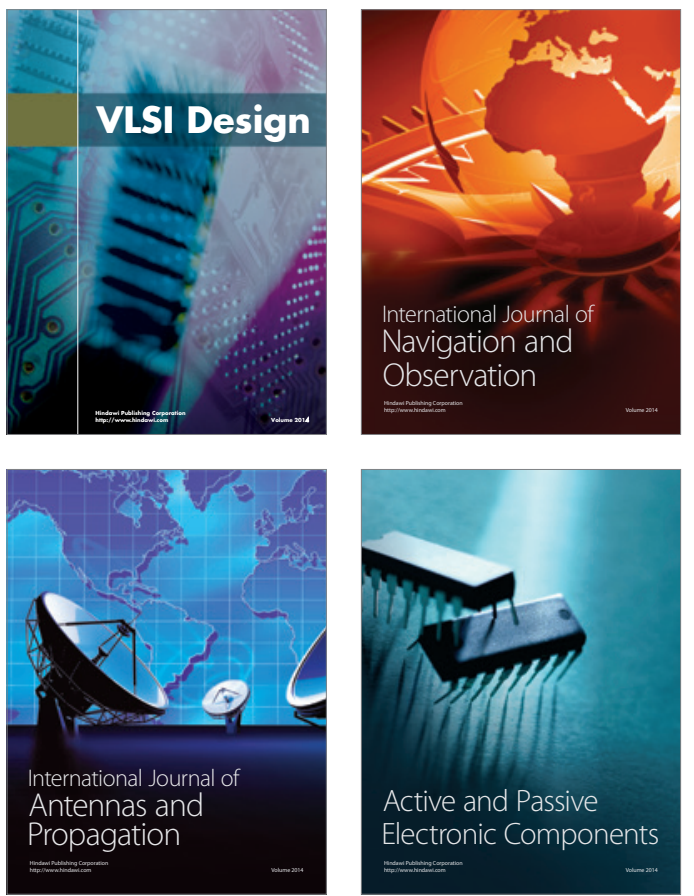
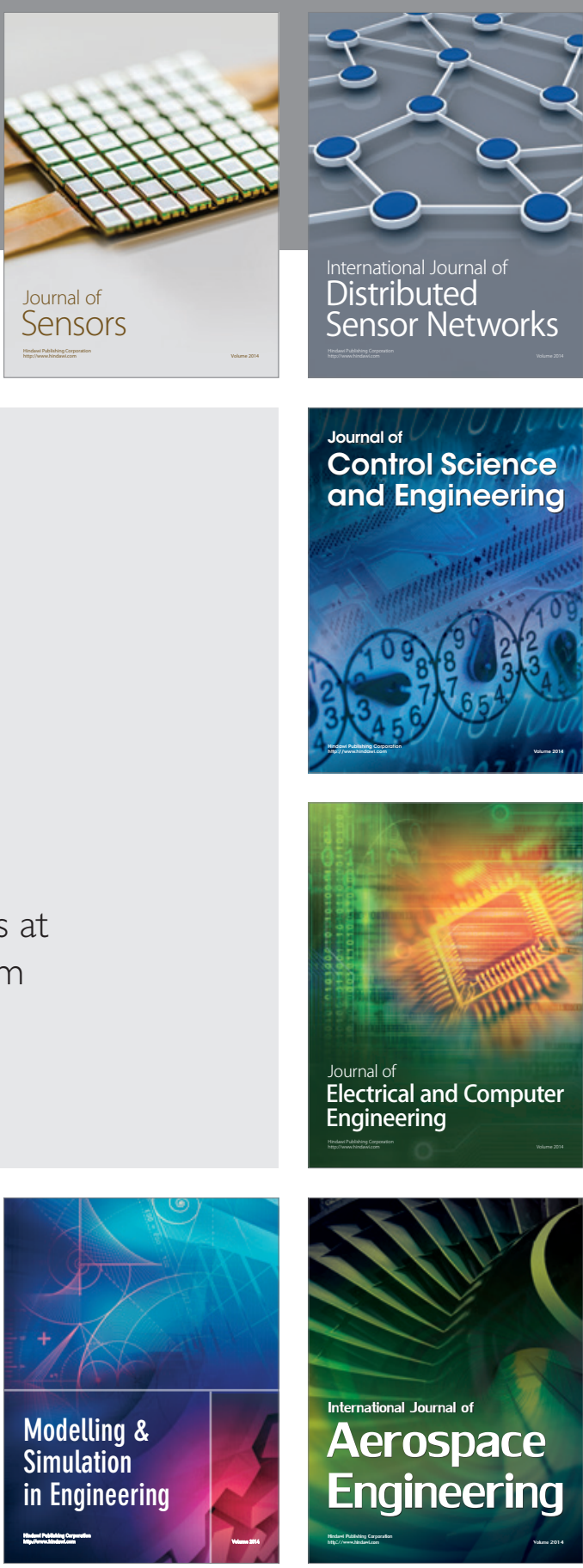

International Journal of

Distributed

Sensor Networks

Journal of

Control Science

and Engineering
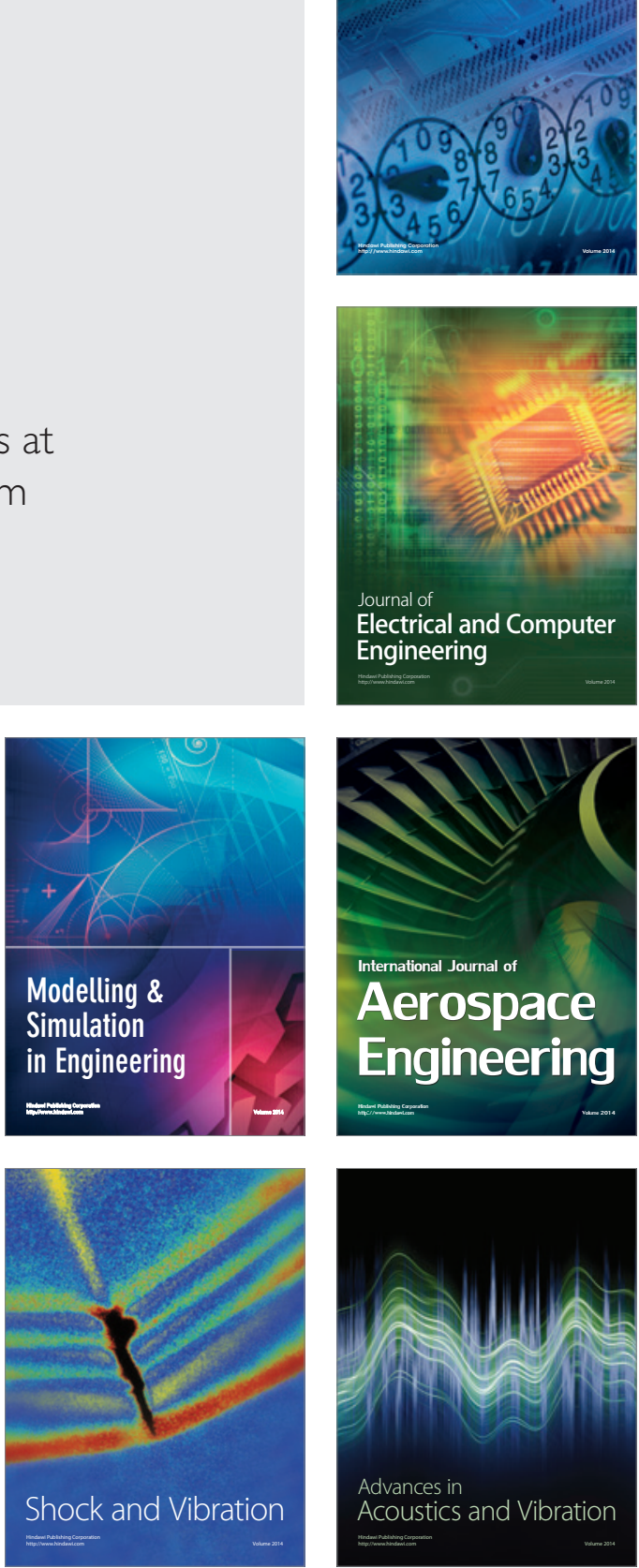